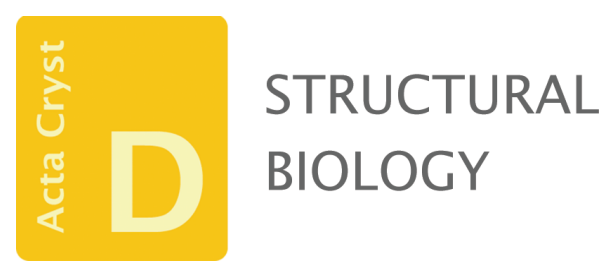

Volume 74 (2018)

Supporting information for article:

Rational modification of vanillin derivatives to stereospecifically destabilize sickle hemoglobin polymer formation

Tanvi M. Deshpande, Piyusha P. Pagare, Mohini S. Ghatge, Qiukan Chen, Faik N. Musayev, Jurgen Venitz, Yan Zhang, Osheiza Abdulmalik and Martin K. Safo 


\section{S1. Synthesis of Compounds}

All reagents used in the syntheses were purchased from Sigma-Aldrich (St. Louis, MO) and ThermoFisher Scientific (Waltham, MA) and utilized without additional purification. Melting points were determined on a Fisher-Scientific melting point apparatus (Serial\# 410N0117), and were uncorrected. ${ }^{1} \mathrm{H}-\mathrm{NMR}$ and ${ }^{13} \mathrm{C}-\mathrm{NMR}$ spectra were obtained on a BPLker $400 \mathrm{MHz}$ spectrometer and tetramethylsilane (TMS) was used as an internal standard. Peak positions were given in parts per million $(\delta)$. Column chromatography was performed on silica gel (grade 60 mesh; Bodman Industries, Aston, PA). Routine thin-layer chromatography (TLC) was performed on silica gel GHIF plates $(250 \mu \mathrm{m}, 2.5$ x $10 \mathrm{~cm}$; Analtech Inc., Newark, DE). MS spectra were obtained from a Perkin Elmer Flexar UHPLC with AxION 2 Time of Flight (TOF) Mass Spectrometer, and the molecular weight of the compounds was within $0.05 \%$ of calculated values. Infrared spectra were obtained on a Thermo Nicolet iS10 FT-IR. Purity of the compounds was determined by HPLC using a Varian Microsorb 100-5 C18 column (250 x 4.6 mm), using Prostar $325 \mathrm{UV}-\mathrm{Vis}(254 \mathrm{~nm})$ as the detector.

\section{S1.1. Synthesis of 2-((6-(Hydroxymethyl)pyridin-2-yl)methoxy)-5-methoxybenzaldehyde, TD-7}

Compound TD-7 was prepared according to standard procedures for similar compounds.(Nnamani et al., 2008) A mixture of 2-hydroxy-5-methoxybenzaldehyde (0.20 mL, $1.5 \mathrm{mmol})$, 6(bromomethyl)-2-pyridinemethanol $(0.30 \mathrm{~g}, 1.5 \mathrm{mmol})$, and $\mathrm{K}_{2} \mathrm{CO}_{3}(0.25 \mathrm{~g}, 1.8 \mathrm{mmol})$ in anhydrous DMF (20 mL) was allowed to stir at room temperature for $12 \mathrm{~h}$. The reaction mixture was diluted with EtOAc $(20 \mathrm{~mL})$, washed with $\mathrm{H}_{2} \mathrm{O}(2 \times 20 \mathrm{~mL})$, brine $(2 \times 10 \mathrm{~mL})$, dried over $\mathrm{Na}_{2} \mathrm{SO}_{4}$ and evaporated under reduced pressure to yield a crude product. The residue was purified by column chromatography (hexanes/EtOAc; 1:1) to yield 0.32g (78\%) of TD-7 as a yellow-

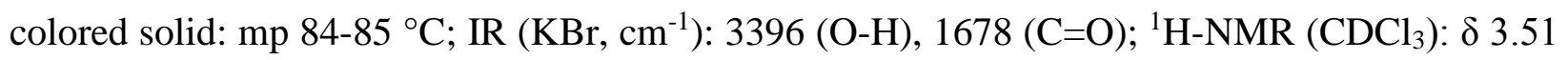
(s, 1H, OH), $3.81\left(\mathrm{~s}, 3 \mathrm{H}, \mathrm{OCH}_{3}\right), 4.79\left(\mathrm{~s}, 2 \mathrm{H}, \mathrm{CH}_{2}\right), 5.29\left(\mathrm{~s}, 2 \mathrm{H}, \mathrm{CH}_{2}\right), 6.99(\mathrm{~d}, J=9.0 \mathrm{~Hz}, 1 \mathrm{H}$, ArH), 7.09-7.13 (dd, $J=9.0,3.2 \mathrm{~Hz}, 1 \mathrm{H}, \mathrm{ArH}), 7.21(\mathrm{~d}, J=7.7 \mathrm{~Hz}, 1 \mathrm{H}, \operatorname{ArH}), 7.37$ (d, $J=3.2 \mathrm{~Hz}$, 1H, ArH), 7.43 (d, J = $7.6 \mathrm{~Hz}, 1 \mathrm{H}, \mathrm{ArH}), 7.74$ (t, 1H, ArH), 10.58 (s, 1H, CHO); ${ }^{13} \mathrm{C}-\mathrm{NMR}$ $\left(\mathrm{CDCl}_{3}\right): \delta 189.24,158.80,155.61,154.17,154.17,137.66,125.56,123.40,119.84,119.64$ 
114.92, 110.917, 71.65, 64.02, 55.86. MS (ESI) $m / z$ found 274.15 $(\mathrm{M}+\mathrm{H})^{+}, 296.14(\mathrm{M}+\mathrm{Na})^{+}$. The purity of the compound was checked by HPLC and was found to be $97.8 \%$ pure.

\section{S1.2. Synthesis of 2-((6-(Hydroxymethyl)pyridin-2-yl)methoxy)-4-methoxybenzaldehyde, TD-9}

Compound TD-9 was prepared according to literature procedure for a similar compound.(Nnamani et al., 2008) A mixture of 2-hydroxy-4-methoxybenzaldehyde (113 mg, $0.74 \mathrm{mmol})$, 6(bromomethyl)-2-pyridinemethanol (150 mg, $0.74 \mathrm{mmol}$ ), and $\mathrm{K}_{2} \mathrm{CO}_{3}$ (123 $\mathrm{mg}, 0.90 \mathrm{mmol}$ ) in anhydrous DMF $(20 \mathrm{~mL})$ was stirred at room temperature for $12 \mathrm{~h}$. The reaction mixture was diluted with EtOAc $(20 \mathrm{~mL})$, washed with $\mathrm{H}_{2} \mathrm{O}(2 \times 20 \mathrm{~mL})$, brine $(2 \times 10 \mathrm{~mL})$, dried over $\mathrm{Na}_{2} \mathrm{SO}_{4}$ and evaporated under reduced pressure to yield a crude product. The crude was purified by column chromatography (Hexanes/EtOAc; 1:1) to yield $190 \mathrm{mg}$ (94\%) of TD-9 as a yellow-colored solid: mp 104-105 ${ }^{\circ} \mathrm{C}$; IR (KBr, cm $\left.{ }^{-1}\right): 3153(\mathrm{O}-\mathrm{H}), 1599(\mathrm{C}=\mathrm{O}) ;{ }^{1} \mathrm{H}-\mathrm{NMR}\left(\mathrm{CDCl}_{3}\right): \delta 3.58(\mathrm{~s}, 1 \mathrm{H}, \mathrm{OH})$, 3.85 (s, 3H, $\left.\mathrm{OCH}_{3}\right), 4.78\left(\mathrm{~d}, 2 \mathrm{H}, \mathrm{CH}_{2}\right), 5.30\left(\mathrm{~s}, 2 \mathrm{H}, \mathrm{CH}_{2}\right), 6.53$ (d, J = 2.2 Hz, 1H, ArH), 6.60 (d, $J=2.16 \mathrm{~Hz}, 1 \mathrm{H}, \mathrm{ArH}), 7.22(\mathrm{~d}, J=7.68 \mathrm{~Hz}, 2 \mathrm{H}, \mathrm{ArH}), 7.47$ (d, $J=7.76 \mathrm{~Hz}, 2 \mathrm{H}, \operatorname{ArH}), 7.76$ (t, $1 \mathrm{H}, \mathrm{ArH}), 7.85$ (d, $J=8.64 \mathrm{~Hz}, 1 \mathrm{H}, \mathrm{ArH}), 10.43$ (s, $1 \mathrm{H}, \mathrm{CHO}) ;{ }^{13} \mathrm{C}-\mathrm{NMR}\left(\mathrm{CDCl}_{3}\right): \delta 166.16$, 162.27, 158.67, 155.51, 137.74, 131.04, 119.85, 119.69, 118.1, 106.68, 99.16, 70.85, 63.87, 55.66. MS (ESI) $m / z$ found $274.11(\mathrm{M}+\mathrm{H})^{+}, 296.09(\mathrm{M}+\mathrm{Na})^{+}$. The purity of the compound was checked by HPLC and was found to be $96.9 \%$ pure.

\section{S1.3. Synthesis of 3-((6-(Hydroxymethyl)pyridine-2-yl)methoxy)-4-methoxybenzaldehyde, TD-8}

Compound TD-8 was prepared according to literature procedure for a similar compound.(Nnamani et al., 2008) A mixture of 3-hydroxy-4-methoxybenzaldehyde (200 mg, $1.0 \mathrm{mmol}$ ), 6(bromomethyl)-2-pyridinemethanol (152 mg, $1.0 \mathrm{mmol}$ ), and potassium carbonate (165 $\mathrm{mg}, 1.2$ $\mathrm{mmol})$ in anhydrous DMF (20 mL) was stirred at room temperature for $12 \mathrm{~h}$. The reaction mixture was diluted with EtOAc $(20 \mathrm{~mL})$, washed with $\mathrm{H}_{2} \mathrm{O}(2 \times 20 \mathrm{~mL})$, brine $(2 \times 10 \mathrm{~mL})$, dried over $\mathrm{Na}_{2} \mathrm{SO}_{4}$ and evaporated under reduced pressure to yield a crude product. The crude was purified by column chromatography (Hexanes/EtOAc; 1:1) to yield $220 \mathrm{mg}$ (80\%) of TD-8 as a whitecolored solid: mp 120-121 ${ }^{\circ} \mathrm{C}$; IR $\left(\mathrm{KBr}, \mathrm{cm}^{-1}\right): 3176(\mathrm{O}-\mathrm{H}), 1681(\mathrm{C}=\mathrm{O}) ;{ }^{1} \mathrm{H}-\mathrm{NMR}\left(\mathrm{CDCl}_{3}\right): \delta$ $3.65(\mathrm{~s}, 1 \mathrm{H}, \mathrm{OH}), 4.00\left(\mathrm{~s}, 3 \mathrm{H}, \mathrm{OCH}_{3}\right), 4.78\left(\mathrm{~d}, 2 \mathrm{H}, \mathrm{CH}_{2}\right), 5.32\left(\mathrm{~d}, 2 \mathrm{H}, \mathrm{CH}_{2}\right), 7.03(\mathrm{~d}, J=8.72 \mathrm{~Hz}$, 1H, ArH), 7.17 (d, $J=7.68 \mathrm{~Hz}, 1 \mathrm{H}, \mathrm{ArH}), 7.44-7.51$ (m, 3H, ArH), 7.71 (t, 1H, ArH); ${ }^{13} \mathrm{C}-\mathrm{NMR}$ 
$\left(\mathrm{CDCl}_{3}\right): \delta 190.66,155.53,154.95,137.54,130.11,126.88,119.99,119.60,111.92,110.98$, 158.79, 71.40, 63.90, 56.22. MS (ESI) $m / z$ found $274.11(\mathrm{M}+\mathrm{H})^{+}, 296.09(\mathrm{M}+\mathrm{Na})^{+}$. The purity of the compound was checked by HPLC and was found to be $99.5 \%$ pure.

\section{S2. Hemoglobin Modification, Oxygen Equilibrium and Antisickling Studies Using Human Sickle Blood}

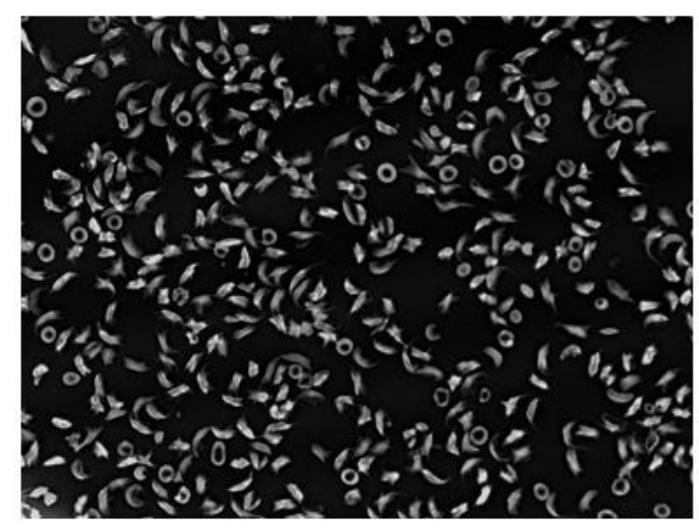

a

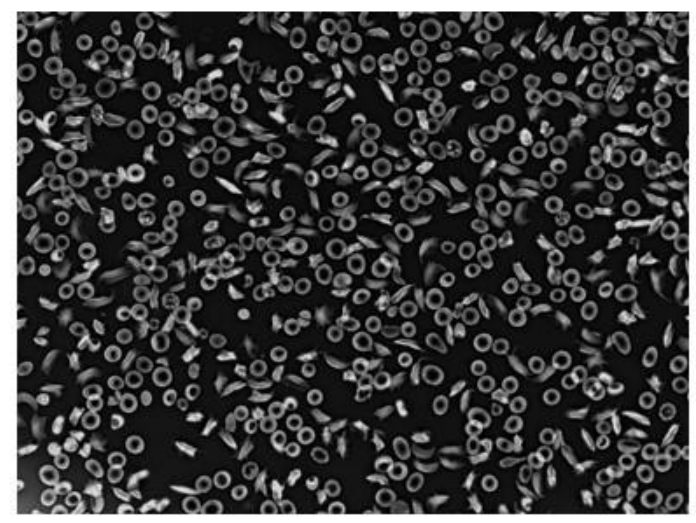

C

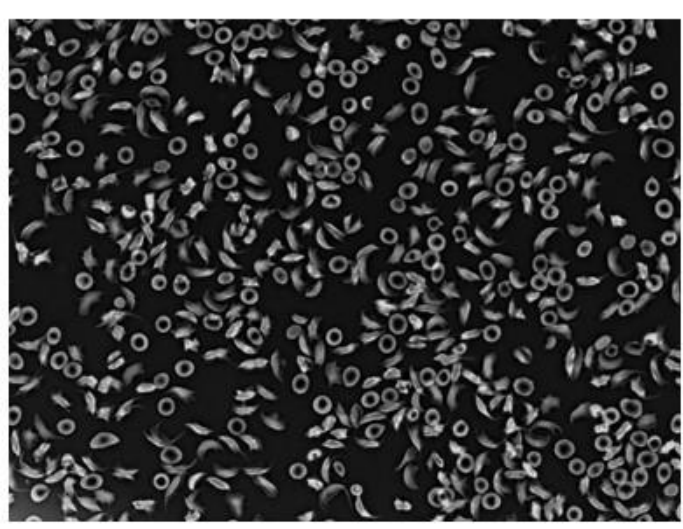

b

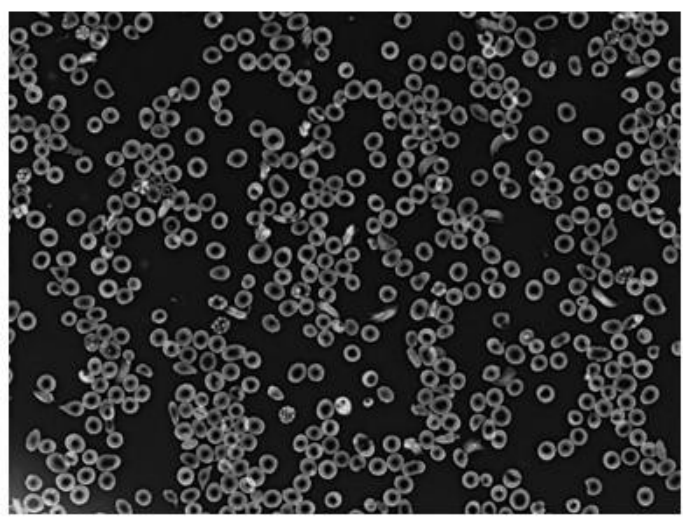

d

Figure S1 Morphology of SS cells with and without various concentrations of TD-7 under $2.5 \%$ oxygen at $37^{\circ} \mathrm{C}$ for $2 \mathrm{hrs}$. (a) Without TD-7. (b, c, d) Upon incubation in the presence of various concentrations of TD-7 $(0.5,1.0$ and $2.0 \mathrm{mM})$, SS cell sickling was prevented in a dose-dependent manner. 


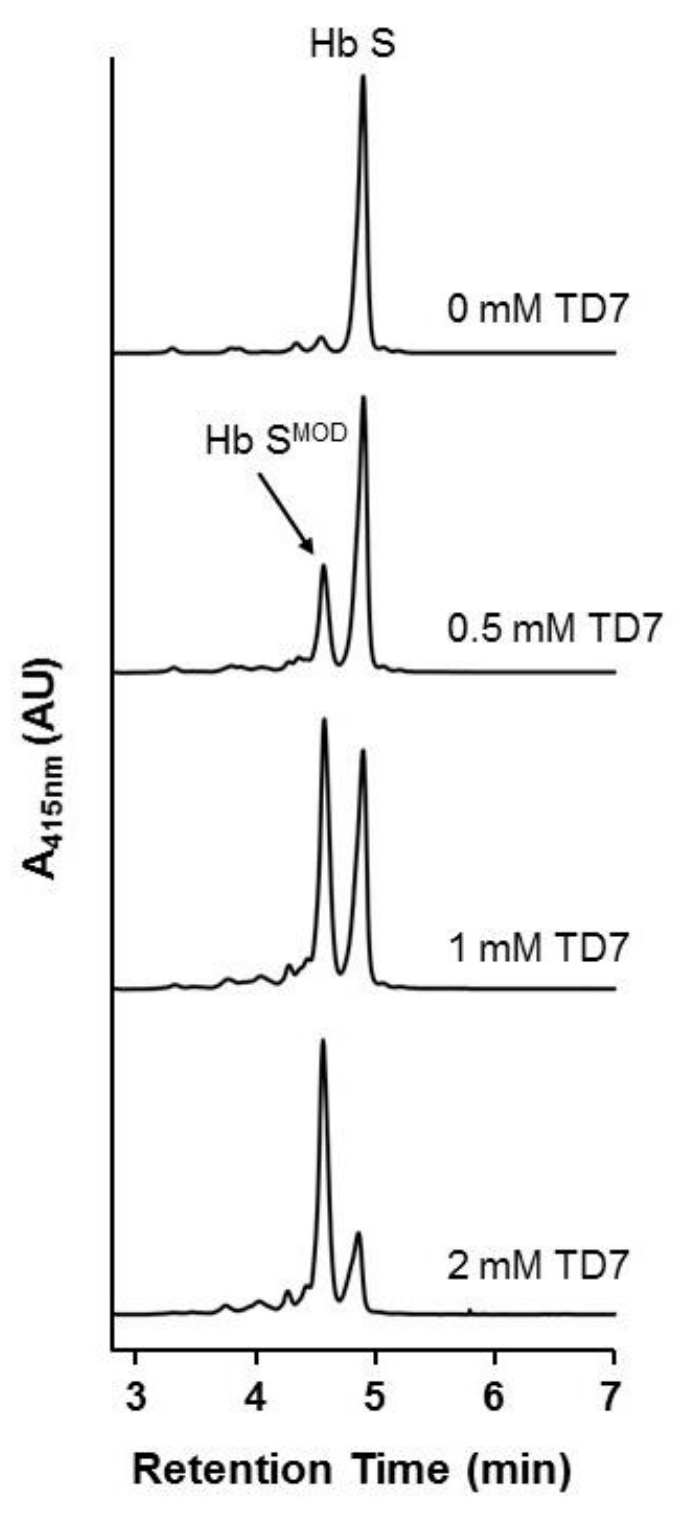

Figure S2 Cation-exchange HPLC patterns of hemoglobin prepared from SS cells that had been preincubated with 0 (control), $0.5,1$, and $2 \mathrm{mM}$ TD-7. 